\title{
AN ALGEBRAIC APPROACH TO THE TOPOLOGICAL DEGREE OF A SMOOTH MAP
}

\author{
BY DAVID EISENBUD ${ }^{1}$
}

The singularities of mappings have attracted a lot of attention lately, perhaps partly because the field touches so many others. However, many elementary problems still remain. I was attracted to the subject myself by a problem shown me by Harold Levine, which I would like to describe. Levine and I worked on this problem together, and the new results that I will discuss come from our joint work, mostly contained in [Eisenbud-Levine].

Topological degree. The problem concerns the computation of the degree of a continuous map

$$
f: M \rightarrow N
$$

between two oriented compact manifolds $M$ and $N$ of the same dimension $n$, written $\operatorname{deg} f$. One way to think of the degree of $f$ is as the number of $f$-preimages of a point in the target manifold $N$. For example, if $f$ is the map given below by "radial projection" from the outer circle to the inner one,

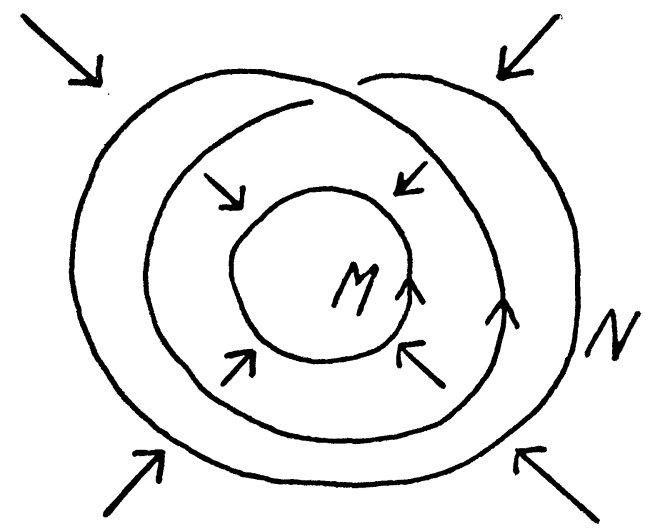

(the arrow heads on $M$ and $N$ represent the orientations)

then every point has two preimages, so the degree of the map is 2.

Of course, care must be taken with maps like the one in the following picture, where again $f$ is given by "radial projection" from the outer circle to the inner:

An Invited Address given at the 83rd Annual Meeting of the American Mathematical Society on January 30, 1977 at St. Louis, Missouri; received by the editors August 12, 1977.

AMS (MOS) subject classifications (1970). Primary 55C25, 14B99; Secondary 10C05, 13B15, 26 A93.

'The author is grateful for the partial support of the NSF during the preparation of this report. 


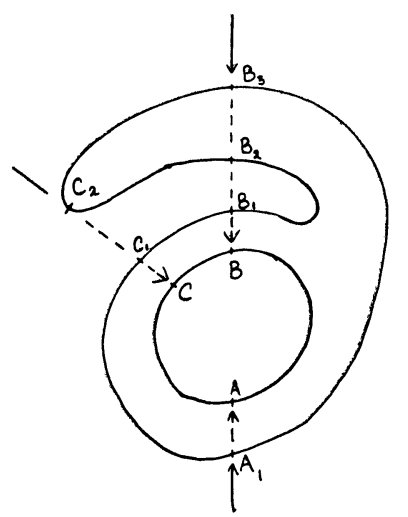

Here point $A$ has 1 preimage $A_{1}$ so the map ought to have degree 1 . But point $B$ has 3 preimages! In order to reduce this number to 1 , we count a preimage point $B_{i}$ as +1 if the map $f$ preserves the orientation near $B_{i}$, and -1 if the map $f$ reverses the orientation near $B_{i}$. Thus $B_{1}$ and $B_{3}$ count positively and $B_{2}$ counts negatively, for an algebraic total of 1 preimage; again, $\operatorname{deg} f=1$.

What about the preimage of $C$ in $N$ ? It consists of the 2 points $C_{1}$ and $C_{2}$. Near $C_{1}, f$ looks the same as near $B_{1}$ or $A_{1}$ so $C_{1}$ can reasonably be counted as +1 preimage. Therefore, $C_{2}$ had better count as no preimages at all! This is not so unreasonable, since $C_{2}$ may be thought of as the point where the positive and negative preimages $B_{2}$ and $B_{3}$ coalesce as $B$ moves to $C$ in $N$.

The above suggests the feasibility of defining the degree rigorously by "appropriately" counting preimages, but it is perhaps reassuring to recall that there is a definition which avoids all such finagling. This definition relies on the fact that the $n$-dimensional integral homology of an oriented compact connected $n$-manifold is canonically isomorphic to the group $Z$ of integers. Thus $f$ induces a map

$$
f_{*}: Z \cong H_{n}(M) \rightarrow H_{n}(N) \cong Z .
$$

Any map from $Z$ to $Z$ is multiplication by some integer, and we may define $\operatorname{deg} f$ as the integer thus associated to $f_{*}$. This definition even shows that homotopic maps have the same degree (some of the significance of the degree is the frequency with which the converse is true, as in the case, for example, when $N$ is the $n$-sphere).

Even given this global definition of the degree, it is still important for computations and applications to give a definition of the degree by counting preimages-that is, to give a definition of the local degree $\operatorname{deg}_{x}$ of $f$ at a point $x \in M$ which is isolated in $f^{-1} f(x)$, in such a way that

$$
\operatorname{deg} f=\sum_{x \in f^{-1}(y)} \operatorname{deg}_{x} f
$$

for any $y \in N$ such that $f^{-1}(y)$ is a finite set. While it is not hard to do this in the above generality (see, for instance, [Milnor (2)]), a more illuminating description is possible if we restrict ourselves to a smaller class of maps and manifolds, so we will henceforth assume that $M$ and $N$ are differentiable manifolds, and that $f$ is continuously differentiable. Since we only need to say how to count a single preimage, $x$, we may (by choosing coordinate 
neighborhoods near $x$ and $f(x)$ ), assume that $f$ is actually a map from a neighborhood $U$ of the origin in $\mathbf{R}^{n}$ to $\mathbf{R}^{n}$ carrying the origin 0 in $U$ to the origin, and that $x=0$. We will indicate that $f$ is such a map by writing

$$
f:\left(\mathbf{R}^{n}, 0\right) \rightarrow\left(\mathbf{R}^{n}, 0\right) .
$$

Choosing coordinates in the target, we may regard $f$ as given by $n$ component maps $f_{1}, \ldots, f_{n}:\left(\mathbf{R}^{n}, 0\right) \rightarrow(\mathbf{R}, 0)$. If we now choose coordinates $X_{1}, \ldots, X_{n}$ in the source, we may define the Jacobian determinant

$$
g=\operatorname{det}\left(\partial f_{i} / \partial x_{j}\right),
$$

which we regard as a function $g:\left(\mathbf{R}^{n}, 0\right) \rightarrow \mathbf{R}$. Any choice of an ordered system of coordinates implies the choice of an orientation, and if the choice of coordinates in source or target is changed in a way that changes the orientation, for example by interchanging two of the coordinates, the function $g$ will change sign. Thus $g$ may be regarded as expressing information on the relative orientations of the source and target of $f$.

The degree at regular and singular points. Recall that a point $x$ in the source of $f$ is said to be regular for $f$ if $g(x) \neq 0$, and that in this case the nonsingular linear mapping given by the matrix

$$
\left(\partial f_{i} / \partial x_{j}\right)
$$

is topologically equivalent to $f$ near 0 , by the inverse function theorem. Since a nonsingular linear map preserves or reverses the orientation of its source according as its determinant is positive or negative, we must have

$$
\operatorname{deg}_{0} f=\operatorname{sgn} g(0)
$$

if 0 is a regular point for $f$. It is not hard to show that this definition is "right" in the sense that, if we return to a map $f$ of compact orientable manifolds, formula (*) will be true for any $y$ in the target all of whose preimages are regular points of $f$-that is, for any $y$ which is a regular value of $f$.

If 0 is not a regular point of $f$, but is isolated in the set $f^{-1}(0)$, then we can try to define the degree at 0 by moving slightly away from 0 to a regular value. Such regular values near 0 always exist (by the theorem of Sard and Brown, almost every point in the target is a regular value), so we may make the above precise by saying that there is a small neighborhood $V$ of 0 in the source of $f$ so that the number

$$
\sum_{x \in V \cap f^{-1}(y)} \operatorname{sgn} g(x)
$$

is independent of $y$, so long as $y$ is a regular value of $f$ and is sufficiently near 0 . Clearly, $\operatorname{deg}_{0} f$ must be this number if formula $(*)$ is to remain true for singular as well as regular values $y$ (the reader who wishes to see these things in more detail should consult [Milnor, (1)]).

It is clear from the above that if two maps $f, g:\left(\mathbf{R}^{n}, 0\right) \rightarrow\left(\mathbf{R}^{n}, 0\right)$ agree on some small neighborhood of 0 , then $\operatorname{deg}_{0} f=\operatorname{deg}_{0} g$. If we identify mappings that agree on sufficiently small neighborhoods of the origin to form equivalence classes, called the germs at 0 of the mappings, the above remark may be restated thus: The number $\operatorname{deg}_{0} f$ depends only on the germ of $f$ at 0 . 
However, the definition of $\operatorname{deg}_{0}(f)$ by $(* *)$ that we have adopted really depends on the choice of a representative mapping $f$ for the germ, since it depends on the action of $f$ at points other than 0 . This brings me, at last, to the problem I want to describe:

Can one calculate the degree of a germ without choosing a representative mapping?

It is worth remarking that Levine and I were not the only ones to consider this problem; Arnold seems to have posed something like it to Zakaljukin, who succeeded in showing that the degree of a map could be deduced from the values of certain polynomials, applied to finitely many of the coefficients in the Taylor series of functions representing $f$ [Zakaljukin]. His proof, however, does not construct these polynomials!

Before trying to solve our problem, we must first consider what invariants of the germ of $f$ are available. The most promising for our purpose seems to be Mather's "local ring".

The local ring of a germ. I will now change notation a little, writing $f$ for the germ of a map $\left(\mathbf{R}^{n}, 0\right) \rightarrow\left(\mathbf{R}^{n}, 0\right)$, and $\operatorname{deg} f$ for the degree of the map at 0 (the only point at which $f$ has a value!). As before, when we restricted ourselves to differentiable germs, there will be a payoff if we add some hypotheses on $f$. For the moment, we simply assume that $f$ is infinitely differentiable.

To attack the problem above, we can make use of an invariant of the germ $f$ which has been studied recently by John Mather, presumably because of its close analogy with a venerable construction in algebraic geometry. This is the local ring $Q(f)$ of $f$, defined by

$$
Q(f)=C^{\infty}\left(\mathbf{R}^{n}, 0\right) /\left(f_{1}, \ldots, f_{n}\right),
$$

where $C^{\infty}\left(\mathbf{R}^{n}, 0\right)$ is the algebra of germs at $0 \in \mathbf{R}^{n}$ of infinitely differentiable real valued functions, and $\left(f_{1}, \ldots, f_{n}\right)$ is the ideal of $C^{\infty}\left(\mathbf{R}^{n}, 0\right)$ generated by the components $f_{1}, \ldots, f_{n}$ of the germ $f$.

The construction of $Q(f)$ does not require a choice of representative mapping for $f$, and it turns out that $Q(f)$ does not depend on the coordinates which were chosen to define the components $f_{1}, \ldots, f_{n}$, either. We will try to calculate the degree of $f$ in terms of $Q(f)$.

But does $Q(f)$ contain enough information? That is, is $\operatorname{deg} f$ really an invariant of $Q(f)$ ? Of course, $Q(f)$ does not contain any information on the relative orientation of the source and target of $f$, so the best we can hope is that $Q(f)$ contains enough information to calculate the absolute value $|\operatorname{deg} f|$. I don't know whether it does or not, but one should perhaps suspect that it doesn't, since $\operatorname{deg} f$ is defined only if 0 is isolated in $f^{-1}(0)$ (for some and hence every representative mapping for $f)$, but $Q(f)$ is always defined, and there is to my knowledge no way of telling from $Q(f)$ whether or not 0 is isolated in $f^{-1} f(0)$. Thus we had better look first for some condition on $Q(f)$ that will guarantee that $\operatorname{deg} f$ is defined.

Finiteness. The best choice for such a property seems to finiteness:

Definition. The germ $f$ is finite if $\operatorname{dim}_{\mathbf{R}} Q(f)<\infty$.

Finiteness is a good property because, on the one hand, nearly all map 
germs have it (in fact, Tougeron has shown that the finite germs form the complement of an algebraic set of infinite codimension in the space of all $C^{\infty}$ germs), while on the other hand it guarantees a close connection between $Q(f)$ and the properties of representative maps of $f$, as the following simple proposition of Mather's shows.

Proposition 1 [MATHeR]. Let $f$ and $g$ be two $C^{\infty}$ mappings $\mathbf{R}^{n} \rightarrow \mathbf{R}^{n}$ carrying the origin to the origin, and suppose that their germs are finite and satisfy $Q(f) \cong Q(g)$ as algebras. Then there exists a neighborhood $U$ of the origin in the source, a diffeomorphism $\sigma$ of $U$, and a smooth family of linear transformations $H_{x}: \mathbf{R}^{n} \rightarrow \mathbf{R}^{n}$ of the target, parametrized by points $x$ in $U$, such that $H_{0}$ is the identity and that for all $x \in U$,

$$
g(x)=H_{x}(f(\sigma(x))) .
$$

This proposition, which almost says that $f$ and $g$ are related by changes of coordinates in the source and target, has as an easy consequence the fact that when $f$ is finite, $\operatorname{deg} f$ is indeed an invariant, up to sign, of the algebra $Q(f)$.

CoROllaRY. If $f, g:\left(\mathbf{R}^{n}, 0\right) \rightarrow\left(\mathbf{R}^{n}, 0\right)$ are finite germs, and if $Q(f) \cong Q(g)$, then $\operatorname{deg} f= \pm \operatorname{deg} g$.

IDEA OF PROOF. By the proposition, representative mappings $f$ and $g$ satisfy $g(x)=H_{x}(f(\sigma(x)))$ near the origin. $H_{x}$, being near the identity, can be ignored in computing the degree [Milnor (2), p. 111]. Since the degree of a composite map is the product of the degrees of the factors, we get

$$
\operatorname{deg} g=\operatorname{deg}(f) \cdot \operatorname{deg} \sigma= \pm \operatorname{deg} f,
$$

since $\sigma$, being a diffeomorphism, has degree \pm 1 .

What is the invariant of $Q(f)$ which gives the degree of $f$ ? This was the problem with which Levine tempted me into this subject. Of course the first thing we did was to look at some examples, hoping eventually to guess a pattern. I would like to show the reader some of the more illuminating of those examples.

Another look at $Q(f)$. It will be helpful in handling the examples to have a different expression for $Q(f)$. To derive this, we use a ring homomorphism about which we teach our students in elementary calculus, the Taylor expansion

$$
\text { Taylor: } C^{\infty}\left(\mathbf{R}^{n}, 0\right) \rightarrow \mathbf{R}\left[\left|x_{1}, \ldots, x_{n}\right|\right],
$$

a homomorphism from the ring of infinitely differentiable germs to the ring of formal power series. The maximal ideal of $C^{\infty}\left(\mathbf{R}^{n}, 0\right)$, the set of germs of functions vanishing at 0 , is generated by the coordinate functions $x_{1}, \ldots, x_{n}$. (Proof: If $f(0)=0$, then $f=\sum_{1}^{n} x_{i} g_{i}(x)$, where $g_{i}(x)=$ $\left.\int_{0}^{1}\left(\partial / \partial x_{i}\right) f\left(t x_{1}, \ldots, t x_{n}\right) d t.\right)$ From this and the fact that $C^{\infty}\left(\mathbf{R}^{n}, 0\right)$ contains the germs of polynomial functions, it follows that if $Q(f)$ is finite dimensional, then the Taylor expansion induces an isomorphism

$$
Q(f) \cong \mathbf{R}\left[\left|x_{1}, \ldots, x_{n}\right|\right] /\left(f_{1}, \ldots, f_{n}\right),
$$

where we have written $f_{i}$ also for the Taylor series of the germ of the $i$ th 
component function of $f$. This expression for $Q(f)$ shows how close we are to the context of algebraic geometry. (In fact, we could even have begun by replacing $f$ by a polynomial mapping: if, for each $i, f_{i}^{\prime}$ agrees with $f_{i}$ up to a sufficiently high order, then $f_{i}-f_{i}^{\prime} \in\left(x_{1}, \ldots, x_{n}\right)\left(f_{1}, \ldots, f_{n}\right)$, so by Nakayama's Lemma,

$$
\left(f_{1}, \ldots, f_{n}\right) \mathbf{R}\left[\left|x_{1}, \ldots, x_{n}\right|\right]=\left(f_{1}^{\prime}, \ldots, f_{n}^{\prime}\right) \mathbf{R}\left[\left|x_{1}, \ldots, x_{n}\right|\right],
$$

whence $Q(f)=Q\left(f^{\prime}\right)$. It is not hard to show directly that $\operatorname{deg} f=\operatorname{deg} f^{\prime}$.)

EXAMPLES. We will begin with the case where the source and target of $f$ have dimension $n=1$. Since we have seen that $|\operatorname{deg} f|$ depends only on $Q(f)=$ $\mathbf{R}[|X|] /(f)$, and since every factor ring of the power series ring in one variable has the form $\mathbf{R}[|X|] /\left(X^{k}\right)$ for some $k$, we will see all the one dimensional possibilities simply by looking at the maps

$$
f(X)=X^{k}, \text { for which } Q(f)=\mathbf{R}[|X|] /\left(X^{k}\right) .
$$

There are two kinds of behavior:

(i) $k$ even. $f$ can be described as a "projection from its graph", as in the following picture:

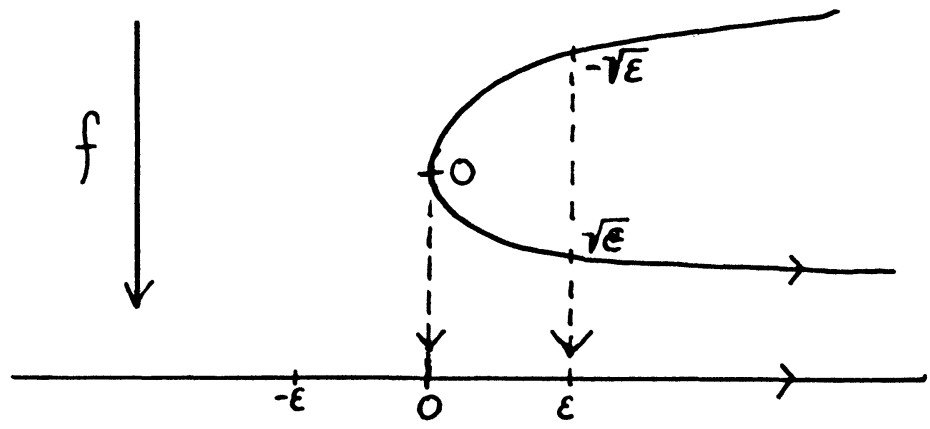

The degree in this case is 0 . To see this, note for example that any $\varepsilon>0$ is a regular value which has 2 preimages; $f$ preserves the orientation near one of them and reverses it at the other. (The lazy will prefer the argument that $-\varepsilon$ has no preimages at all-and is thus automatically regular.)

(ii) $k$ odd. With the same scheme, $f$ "looks" like:

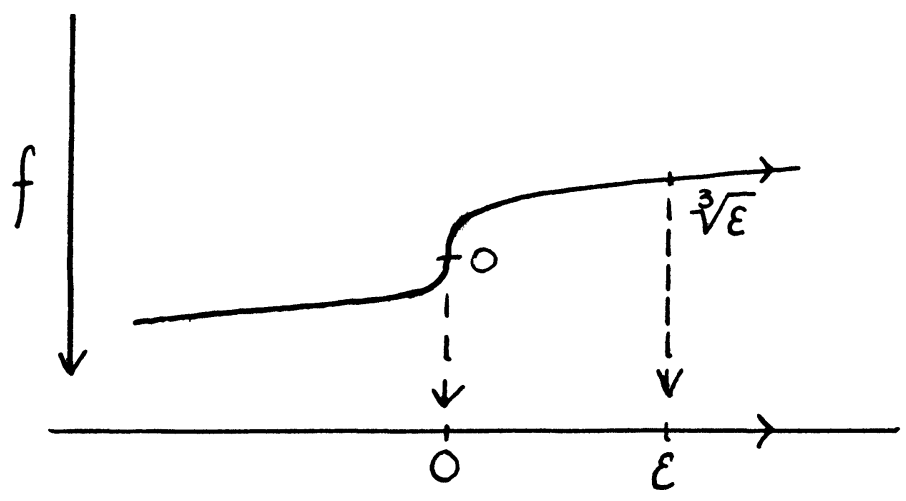


Clearly $\operatorname{deg} f= \pm 1$, the sign depending on the orientations chosen.

Finally an example which is slightly less banal, and so perhaps more helpful:

(iii) Identify $\mathbf{R}^{2}$ with the complex line $\mathbf{C}^{1}$, and consider the map $f$ :

$$
\underbrace{\mathbf{R}^{2}=\mathbf{C}^{1} \stackrel{f_{1}}{\rightarrow} \mathbf{C}^{1}=\mathbf{R}^{2}}_{f}
$$

where $f_{1}(z)=z^{2}$. The two components of $f$ (with respect to the basis 1 , $\sqrt{-1}$ for $C$ ) are $X^{2}-Y^{2}$ and $2 X Y$, so

$$
Q(f)=\mathbf{R}[|X, Y|] /\left(X^{2}-Y^{2}, 2 X Y\right) .
$$

As a basis we may take the classes

$$
\overline{1}, \bar{X}, \bar{Y}, \overline{X^{2}+Y^{2}},
$$

where - denotes reduction modulo $\left(X^{2}-Y^{2}, 2 X Y\right)$. The multiplication is given by

$$
\begin{gathered}
0=\bar{X} \cdot \bar{Y}=\bar{X} \cdot \overline{X^{2}+Y^{2}}=\bar{Y} \cdot \overline{X^{2}+Y^{2}}=\left(\overline{X^{2}+Y^{2}}\right)^{2}, \\
(\bar{X})^{2}=(\bar{Y})^{2}=\frac{1}{2} \overline{X^{2}+Y^{2}} .
\end{gathered}
$$

Using the same scheme as in (i), we may picture $f$ as a projection; it is the familiar two-sheeted covering map of $C$, ramified over 0 .

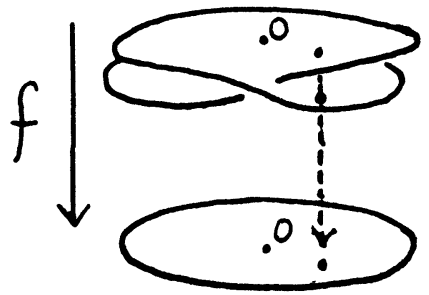

From the picture we see that a regular value near 0 has 2 preimages (any nonzero complex number has 2 square roots!) and that $f$ preserves the canonical orientation (1 first, then $\sqrt{-1}$ ) of $\mathbf{C}$ near each of these. Thus with that orientation, $\operatorname{deg} f=2$.

Other powers of $z$ may be treated similarly. It turns out that, if $f_{1}(z)=z^{k}$, then $\operatorname{dim} Q(f)=k^{2}$, while $\operatorname{deg} f=k$.

The results of Serre and Berger. In examining these examples, Levine and I had in mind an observation of Serre [Bass] and a theorem due essentially to Berger ([Berger]-see also [Scheja-Storch]) which throw some light on the structure of the algebra $Q(f)$. These results-or rather, their applicable special cases-may be summarized thus:

Proposition 2. If $f:\left(\mathbf{R}^{n}, 0\right) \rightarrow\left(\mathbf{R}^{n}, 0\right)$ is a finite germ, then the algebra $Q(f)$ has a unique minimal nonzero ideal. This ideal is one-dimensional over $\mathbf{R}$, and is spanned by the residue class $J$ of the germ $g$ of the Jacobian of $f$.

For instance, in examples (i) and (ii) above, $J$ is the class of $k x^{k-1}$, while in example (iii), $J$ is $4\left(X^{2}+Y^{2}\right)$. Using these facts about the structure of $Q(f)$, 
it is natural to introduce a symmetric bilinear form on $Q(f)$, whose properties reflect the algebra structure, as follows: Choose a real-linear functional $\varphi$ : $Q(f) \rightarrow \mathbf{R}$, and let $\langle a, b\rangle_{\phi}=\varphi(a b)$ for $a, b \in Q(f)$. Since by the proposition $J \neq 0 \in Q(f)$, we may choose $\varphi$ to take a nonzero value on $J$, say $\varphi(J)=1$. The statement that $J$ spans the unique minimal nonzero ideal of $Q(f)$ is easily seen to imply that $\langle,\rangle_{\varphi}$ is nonsingular. (Proof: If $\langle a, Q(f)\rangle_{\varphi}=0$, then $\varphi(a Q(f))=0$. Since $\operatorname{dim}_{R} Q(f)<\infty$, the ideal $a Q(f)$ contains a minimal ideal, and thus by the proposition, contains $J$, unless $a=0$. Since $\varphi(J) \neq 0$, we must have $a=0$.)

Of course real symmetric bilinear forms are much simpler than algebras; nonsingular ones possess, aside from the dimension of the space on which they are defined, a single invariant, called the signature, which we will abbreviate sign: If we express the form as a diagonal matrix with respect to some orthogonal basis, the signature is the sum of the signs of the diagonal entries of the matrix.

The Main Theorem. After some computations Levine and I were convinced of the likelihood of the following result, which we later succeeded in proving:

MAIN TheORem. Let $f:\left(\mathbf{R}^{n}, 0\right) \rightarrow\left(\mathbf{R}^{n}, 0\right)$ be a finite map germ, and let $\varphi$ : $Q(f) \rightarrow \mathbf{R}$ be a linear functional whose value on J, the class of the Jacobian, is 1. Then

$$
\operatorname{deg} f=\operatorname{sign}\langle,\rangle_{\varphi} .
$$

Note that there is no more ambiguity here in the sign of the degree; we have used the class of the Jacobian to recover this information.

With a bit of algebra, it is possible to deduce from this a result which allows one to deduce $|\operatorname{deg} f|$ directly from $Q(f)$ :

COROLlaRY. Let $f:\left(\mathbf{R}^{n}, 0\right) \rightarrow\left(\mathbf{R}^{n}, 0\right)$ be a finite map germ. Let I in $Q(f)$ be an ideal which is maximal with respect to the property $I^{2}=0$. Then

$$
|\operatorname{deg} f|=\operatorname{dim}_{\mathbf{R}} Q(f)-2 \operatorname{dim}_{\mathbf{R}} I .
$$

SKETCH OF THE PROOF OF THE COROLLARY. $I$ turns out to be a maximal isotropic subspace; that is, a subspace of $Q(f)$ maximal with respect to the property $\langle I, I\rangle_{\varphi}=0$. (Since $\langle I, I\rangle_{\varphi}=\varphi\left(I^{2}\right)=\varphi(0)$, it is obvious that $I$ is isotropic. That $I$ is maximal among isotropic subspaces is slightly more complicated.) It follows that $Q(f)$ breaks up as a direct sum of subspaces, of the form

$$
Q(f)=I \oplus I^{*} \oplus D,
$$

where $I^{*}$ is the dual of $I$ with respect to $\langle,\rangle_{\phi}$, and where the restriction of $\langle,\rangle_{\varphi}$ to $D$ is (positive or negative) definite. Thus

$$
\begin{aligned}
\left|\operatorname{sign}\langle,\rangle_{\boldsymbol{}}\right| & =\operatorname{dim}_{\mathbf{R}} Q(f)-\operatorname{dim}_{\mathbf{R}} I-\operatorname{dim}_{\mathbf{R}} I^{*} \\
& =\operatorname{dim}_{\mathbf{R}} Q(f)-2 \operatorname{dim}_{\mathbf{R}} I .
\end{aligned}
$$

To check the Corollary against examples (i) and (ii), note that in the first case the maximal ideal $I$ with $I^{2}=0$ is $x^{k / 2} Q(f)$, which has just half the dimension of $Q(f)$, while in the second case that ideal is $x^{k+1 / 2} Q(f)$, which 
has dimension $(k-1) / 2$. Thus for case (i) the Corollary gives $|\operatorname{deg} f|=0$, and in case (ii) it gives $|\operatorname{deg} f|=1$.

With example (iii), we will illustrate the Main Theorem. We may define $\varphi$ by choosing its values on a basis of $Q(f)$, for example as:

$$
\begin{gathered}
\varphi(\overline{1})=\varphi(\bar{X})=\varphi(\bar{Y})=0, \\
\varphi\left(\overline{X^{2}+Y^{2}}\right)=1 / 4,
\end{gathered}
$$

(Here the value of $\varphi\left(\overline{X^{2}+Y^{2}}\right)$ is chosen to make $\varphi(J)=\varphi\left(4 \overline{X^{2}+Y^{2}}\right)=$ 1.)

With this choice the bilinear form may be easily computed as

\begin{tabular}{r|cccc}
$\langle a, b\rangle_{\varphi}=\varphi(a b)$ & 1 & $\bar{X}$ & $\bar{Y}$ & $\overline{X^{2}+Y^{2}}$ \\
\hline 1 & 0 & 0 & 0 & $1 / 4$ \\
$\bar{X}$ & 0 & $1 / 8$ & 0 & 0 \\
$\bar{Y}$ & 0 & 0 & $1 / 8$ & 0 \\
$\overline{X^{2}+Y^{2}}$ & $1 / 4$ & 0 & 0 & 0
\end{tabular}

Since $\left(\begin{array}{ll}0 & 1 / 4 \\ 1 / 4 & 0\end{array}\right)$ becomes $\left(\begin{array}{ccc}-1 / 4 & 0 \\ 0 & 1 / 4\end{array}\right)$ with respect to an orthogonal basis, this form has a matrix

$$
\left[\begin{array}{cccc}
-1 / 4 & 0 & 0 & 0 \\
0 & 1 / 8 & 0 & 0 \\
0 & 0 & 1 / 8 & 0 \\
0 & 0 & 0 & 1 / 4
\end{array}\right]
$$

with respect to an orthogonal basis of $Q(f)$, so the signature is 2 , the same as the degree of $f$ ! Thus the Theorem and its Corollary are clearly true.

IDEA OF THE PROOF OF THE MAIN THEOREM. To prove the Main Theorem, we must replace the idea of "counting (appropriately) the preimages near 0 of a regular value $y$ as $y$ goes to 0 " by some purely algebraic notion. Fortunately, this sort of problem is well understood in algebraic geometry, and we need only borrow the right tools. To avoid the introduction of sheaves of germs of analytic functions or (almost equivalently) of étale covers, we will assume that $f$ is given by $n$ polynomials $f_{1}, \ldots, f_{n}$ such that

(1) The map of polynomial rings

$$
B=\mathbf{R}\left[Y_{1}, \ldots, Y_{n}\right] \stackrel{F}{\rightarrow} \mathbf{R}\left[X_{1}, \ldots, X_{n}\right]=A
$$

sending $Y_{i} \mapsto f_{i}(X)$ makes $A$ into a finitely generated $B$-module, and

(2) The only preimage of 0 under the map

$$
f_{\mathbf{C}}: \mathbf{C}^{n} \rightarrow \mathbf{C}^{n}
$$

given by the same polynomials $f_{1}, \ldots, f_{n}$ is 0 .

Each of Conditions (1) and (2) is somewhat stronger than the statement that the germ of $f$ at 0 is finite. Note that the restriction to the case of polynomials is not serious, by Proposition 1. Furthermore, we could guarantee that Condition 1 holds, without affecting $Q(f)$ or $\operatorname{deg}_{0} f$, by replacing 
each $f_{i}$ by $f_{i}+x_{i}^{N}$, when $N$ is a sufficiently large integer. But we do not know whether it is always possible to replace $f$ by a mapping for which Condition (2) is satisfied without changing $Q(f)$. Thus the general case of the Main Theorem requires for the moment either the extra machinery already mentioned, or a circuitous argument that uses the polynomial map but separates the preimages near zero from those far away.

With these hypotheses, the notion of the preimage of a point $y=$ $\left(r_{1}, \ldots, r_{n}\right)$ in the target $\mathbf{R}^{n}$ has its algebraic analogue in the algebra

$$
\begin{aligned}
Q_{y}(f) & =\mathbf{R}\left[X_{1}, \ldots, X_{n}\right] /\left(f_{1}-r_{1}, \ldots, f_{n}-r_{n}\right) \\
& =\mathbf{R}[X] /\left(Y_{1}-r_{1}, \ldots, Y_{n}-r_{n}\right) \mathbf{R}[X]
\end{aligned}
$$

called the algebra of the fiber of $F$ over $b$. It is not difficult to show that the local ring of the germ of $f$ at $0, Q(f)$ in our old notation, is just $Q_{0}(f)$ in our new notation.

In fact the existence of a map $Q(f) \rightarrow Q_{0}(f)$ is clear, since we may take germs of polynomials to represent the elements of $Q(f)=$ $C^{\infty}\left(\mathbf{R}^{n}, 0\right) /\left(f_{1}, \ldots, f_{n}\right)$; the fact that the map is an isomorphism follows from the finiteness of $f$ and from Condition (2).

What about the notion of $y$ "going to 0 "? This is expressed by the map $F$ of polynomial rings! For $F$ contains, simultaneously, data for constructing all the fiber algebras $Q_{y}(f)$.

If now $y$ is a regular value of $f$, then it is easy to see that $Q_{y}(f)$ will have the form

$$
\mathbf{R} \times \cdots \times \mathbf{R} \times Q_{y}^{\prime}(f)
$$

where the different copies of $\mathbf{R}$ correspond to the points $X_{1}, \ldots, X_{s}$, the preimage $f^{-1}(y)$, and $Q_{y}^{\prime}(f)$ has only complex residue class fields (corresponding to nonreal pairs of complex conjugate preimages of $y$ under $f_{\mathrm{C}}$ ).

Our hypotheses 1 and 2 imply that the $x_{i}$ all go to 0 as $y$ goes to 0 , so that the degree of $f$ at 0 will be expressed by the formula

$$
\operatorname{deg} f=\sum_{i=1}^{s} \operatorname{deg} x_{i}=\sum_{i=1}^{s} \operatorname{sgn} g\left(x_{i}\right) \text {. }
$$

The only thing now lacking for a proof of the Main Theorem is a smoothly varying family of bilinear forms $\langle,\rangle_{y}$ on $Q_{y}(f)$ such that $\langle,\rangle_{0}=\langle,\rangle_{\varphi}$ and that for a regular value $y$ with preimages $x_{1}, \ldots, x_{s},\langle,\rangle_{y}$ will have signature equal to the sum in $(* *)$; for if $\langle,\rangle_{y}$ changes smoothly, it will remain nonsingular, and thus have constant signature, for $y$ near 0 .

Using bases, it is easy to define a family of forms $\langle,\rangle_{y}$ extending $\langle,\rangle_{0}=\langle,\rangle_{\Phi}$; but unfortunately there seems to be no direct method for computing the signatures of \langle\rangle$_{y}$.

An idea from number theory. A way out of this dilemma is provided by an idea that was, in a special case, familiar to number theorists in the last century. It was extended to the case we need by R. Berger [Berger] in 1962; since then it has become a part of the Grothendieck duality machine (under the general name of "residue") [Beauville]. To see it in its primitive form, let us return to the case $n=1$. 
Consider the extension of polynomial rings $B=\mathbf{R}[Y] \hookrightarrow \mathbf{R}[X]=A$, where $Y$ goes to $f(X)$, as an analogue of an algebraic extension of the integers $Z \subset \mathcal{O}$ where $\mathcal{\theta}$ is the ring of all algebraic integers in some number field $K$, a finite extension of the rational numbers. In our case, $A$ is generated as a $B$-algebra by a single element $X$, so we will assume that $\theta=Z[\alpha]$ for some algebraic integer $\alpha$. Let $\alpha$ satisfy the minimal polynomial equation

$$
g(\alpha)=\alpha^{m}+r_{1} \alpha^{m-1}+\cdots+r_{m}=0, \quad r_{i} \in Z .
$$

Note that $X$ satisfies the equation $f(X)-Y=0$, where $f$ has coefficients in $\mathbf{R} \subset \boldsymbol{B}$, so the analogue of $g$ in our polynomial-ring case is $f(X)-Y$, considered as a polynomial in $X$ with coefficients in $B=\mathbf{R}[Y]$. Note that, as such, $f(X)-Y$ differs from $f(X)$ only by the term $Y$ which is "constant" (that is, in $B$ ).

In the number theoretic case, a primary concern is the factorization in $\theta$ (perhaps into prime ideals) of primes in $Z$, and in this a special role is played by prime ideals $P$ of $\mathcal{O}$ whose squares contain primes of $Z$. The classical result (due to Dedekind) is that $P$ contains the square of a prime integer if and only if $P$ contains the element $g^{\prime}(\alpha)$, where $g^{\prime}$ denotes the usual derivative. The route to a proof of this fact passes by an explicit computation of the $Z$ dual of $\theta$; it turns out that the $Z$-linear maps from $\theta$ to $Z$ are exactly the maps $\mathcal{\theta} \exists r \rightarrow \operatorname{Tr}\left(r s / g^{\prime}(d)\right)$ for arbitrary $s \in \mathcal{O}$, where $\operatorname{Tr}$ denotes the trace map from the quotient field $K$ of $\mathcal{\theta}$ to the field of rational numbers $Q \cdot(\operatorname{Tr}(\beta)$ may be defined as the trace of the $Q$-linear transformation of $K$ given by multiplication by $\beta$ on $K$.) Note that, while $\operatorname{Tr}$ carries $\theta$ to $Z$ for more or less trivial reasons, it is not obvious that the trace of $r s / g^{\prime}(\alpha)$-which is in $K$ but not necessarily in $\mathcal{\theta}$-will be in $Z$ ! A beautiful exposition of the proof of the above part may be found in Serre's book [Serre, Chapter III]; suffice it here to say that the proof rests on another result that we often teach to our calculus students, Euler's formula for "partial fractions":

$$
\frac{1}{g(X)}=\sum_{\alpha \text { a root of } g} \frac{1}{g^{\prime}(\alpha)(X-\alpha)} .
$$

In a sense, the heart of this number-theoretic development is the construction of the distinguished $Z$-linear functional $T: \mathcal{O} \rightarrow Z: r \mapsto$ $\operatorname{Tr}\left(r / g^{\prime}(\alpha)\right)$ (depending, of course, on the choice of $\alpha$ ) and the statement that every functional $\mathcal{O} \rightarrow Z$ is in some sense a multiple of $T$.

In our case, $\mathbf{R}[Y] \hookrightarrow \mathbf{R}[X]$, we can get a distinguished $\mathbf{R}[Y]$-linear functional by an analogous formula:

$$
T: \mathbf{R}[X] \rightarrow \mathbf{R}[Y]: p(X) \mapsto \operatorname{Tr}\left(\frac{p(X)}{f^{\prime}(X)}\right),
$$

where $\operatorname{Tr}$ is now the trace associated with the field extension $\mathbf{R}(Y) \subset \mathbf{R}(X)$, and where we have used $f^{\prime}(X)$ instead of the derivative of the analogue of $g$, because $f$ differs from that analogue by a constant in the appropriate sense.

Berger's remarkable discovery was that, although no one knows an analogue of Euler's formula for functions of many variables, the above functional does have natural generalization to the case of many variables. If 


$$
\mathrm{R}\left[Y_{1}, \ldots, Y_{n}\right] \stackrel{F}{\rightarrow} \mathrm{R}\left[X_{1}, \ldots, X_{n}\right]
$$

is a map satisfying our Condition (1) of finiteness, then the functional is obtained merely by substituting the Jacobian for the derivative in the above definition of $T$. That is, if

$$
g=\operatorname{det}\left(\frac{\partial f_{1} \cdots f_{n}}{\partial X_{1} \cdots X_{n}}\right) \in \mathbf{R}\left[X_{1}, \ldots, X_{n}\right]
$$

is the Jacobian determinant, then there is an $R\left[Y_{1}, \ldots, Y_{n}\right]$-linear functional

$$
T: \mathbf{R}\left[X_{1} \cdots X_{n}\right] \rightarrow \mathbf{R}\left[Y_{1}, \ldots, Y_{n}\right]: p(X) \mapsto \operatorname{Tr}\left(\frac{p(X)}{g}\right)
$$

and as before, every such functional is a multiple of $T$ in an appropriate sense.

Completion of the proof of the Main Theorem. To finish the proof of the Main Theorem, one first notes that $T$ induces an R-linear functional $Q_{y}(f)$ $\rightarrow{ }^{T_{1}} \mathbf{R}$ for each $y$, by reduction modulo the ideal $\left(Y_{1}-r_{1}, \ldots, Y_{n}-r_{n}\right)$ where the $r_{i}$ are the coordinates of $y$ : More precisely,

$$
\begin{aligned}
T_{y} & =T \otimes_{\mathbf{R}[Y]} \mathbf{R}: \mathbf{R}[X] \otimes_{\mathbf{R}[Y]} \mathbf{R}[Y] /\left(Y_{1}-r_{1}, \ldots, Y_{n}-r_{n}\right) \\
& \rightarrow \mathbf{R}[Y] \otimes_{\mathbf{R}[Y]} \mathbf{R}[Y] /\left(Y_{1}-r_{1}, \ldots, Y_{n}-r_{n}\right) \\
& =\mathbf{R} .
\end{aligned}
$$

Also, $T_{0}(J)=\operatorname{Tr}(g / g)=\operatorname{Tr}(1)=k$ is the degree of the field extension $\mathbf{R}(X) / \mathbf{R}(Y)$, a positive integer. Thus $\varphi_{0}=(1 / k) T_{0}$ satisfies the hypothesis of the Main Theorem.

Of course it is an obvious consequence of the Main Theorem that the signature of the form $\langle,\rangle_{\varphi}$ does not depend on $\varphi$ so long as $\varphi(J)=1$. But this is easy to prove directly; for if $\varphi^{\prime}$ is another functional with $\varphi^{\prime}(J)=1$, then $\varphi_{t}=t \varphi+(1-t) \phi^{\prime}$ is a whole 1-parameter family of such functionals. By Proposition 2, $\langle,\rangle_{q}$ is nonsingular for every $t$, so the signature is independent of $t$. Thus it suffices to prove the Main Theorem for $\varphi=\varphi_{0}=$ $(1 / k) T_{0}$.

Defining $\langle a, b\rangle_{y}=(1 / k) T_{y}(a b)$ for $a, b \in Q_{y}(f)$, we get a family of bilinear forms of the kind we desire. Since $\langle,\rangle_{y}$ is defined through the multiplication in $Q_{y}(f)$, the decomposition of $Q_{y}(f)$ for a regular value $y$ given in formula (*) will be an orthogonal decomposition of $\langle,\rangle_{y}$. Thus we need only compute the signature of $\langle$,$\rangle , restricted to each factor in (*).$

Let us deal first with the factor $Q_{y}^{\prime}(f)$. Since the residue class fields of this algebra are all isomorphic to $\mathbf{C}$, we may regard it as a $\mathbf{C}$-algebra. Let $\varphi^{\prime}$ : $Q_{y}^{\prime}(f) \rightarrow \mathbf{R}$ be the functional obtained by restricting $T_{y}$. For any real number $\theta, \varphi_{\theta}^{\prime}(a)=\varphi^{\prime}\left(e^{i \theta} a\right)$ is a real linear functional, and since $\langle,\rangle_{\varphi^{\prime}}$ is nonsingular and $e^{i \theta}$ is a unit in $Q_{y}^{\prime}(f)$, the functionals $\langle,\rangle_{\phi^{\prime}}$ will all be nonsingular and thus will all have the same signature. But $\langle,\rangle_{\phi_{\phi}^{\prime}}=-\langle,\rangle_{\phi^{\prime}}$, so sign $\langle,\rangle_{\phi^{\prime}}=$ sign $\langle,\rangle_{\phi_{m}^{\prime}}=-$ sign $\langle,\rangle_{\phi^{\prime}}$. Thus sign \langle\rangle$_{\phi^{\prime}}=0$, and the factor $Q_{y}^{\prime}(f)$ contributes nothing to the signature of $\langle,\rangle_{y}$.

Finally, we compute the signature of $\langle$,$\rangle , restricted to the factor \mathbf{R}$ 
$\times \cdots \times \mathbf{R}$ of $Q_{y}(f)$. Since $y$ is a regular value, the numbers $g\left(x_{i}\right)$ are nonzero, so the elements

$$
e_{i}=\left(0, \ldots, g\left(x_{i}\right), \ldots, 0\right) \in \mathbf{R} \times \cdots \times \mathbf{R}
$$

form an orthogonal basis for $\mathbf{R} \times \cdots \times \mathbf{R}$ with respect to the form $\langle,\rangle_{y}$. We need only compute $\left\langle e_{i}, e_{i}\right\rangle_{y}$ :

$$
\begin{aligned}
\left\langle e_{i}, e_{i}\right\rangle_{y} & =(1 / k) T_{y}\left(\left(0, \ldots, g\left(x_{i}\right), \ldots, 0\right)^{2}\right) \\
& =(1 / k) T_{y}\left(\left(0, \ldots, g\left(x_{i}\right)^{2}, \ldots, 0\right)\right) \\
& =(1 / k) g\left(x_{i}\right) T_{y}\left(\left(0, \ldots, g\left(x_{i}\right), \ldots, 0\right)\right) .
\end{aligned}
$$

But $g\left(x_{i}\right)$ is the residue class in the $i$ th factor of $\mathbf{R} \times \cdots \times \mathbf{R}$ of $g$, so the value of the last expression is the value of the function

$$
(1 / k) g\left(x_{i}\right) \cdot T(g)
$$

at $x_{i}$.

However $T(g)=\operatorname{Tr}(g / g)=k$ is the constant function with value $k$, so we get $\left\langle e_{i}, e_{i}\right\rangle=g\left(x_{i}\right)$.

Thus the signature of $\langle,\rangle_{y}$, which is the sum of the signs of $\left\langle e_{i}, e_{i}\right\rangle$, is the sum of the signs of the Jacobian of $f$ at the preimages near 0 of a regularvalue of $f$, which was our definition of the degree of $f$.

\section{Some remaining questions.}

(1) One is: What about other relative dimensions? For example, if one considers a finite map germ $f:\left(\mathbf{R}^{n}, 0\right) \rightarrow\left(\mathbf{R}^{p}, 0\right)$, with $p>n$, then $Q(f)$ will no longer satisfy the Serre-Berger Proposition, but there will be a sort of family of bilinear forms on $Q(f)$, corresponding to the different minimal ideals. Is there topologically interesting information to be had from this family? What about the degrees of maps obtained from $f$ by projecting $\mathbf{R}^{p} \rightarrow \mathbf{R}^{n}$ in a generic way. (One gets in that way a different family of bilinear forms.)

As for the case $p<n$, one obtains a ring which is the analogue of $Q(f)$, but has higher Krull dimension. Do the invariants of such rings (Hilbert function ...) have any topological significance? One might also try to do something with the germs derived from $f$ by composing with generic inclusions $\mathbf{R}^{p} \rightarrow \mathbf{R}^{n}$; their local rings have properties closely related to the properties of this local ring of $f$.

(2) A second question that fits naturally into the framework of this report is the one examined recently in [Damon-Galligo]: What is the significance for a map germ $f:\left(\mathbf{R}^{n}, 0\right) \rightarrow\left(\mathbf{R}^{n}, 0\right)$, of the number $\operatorname{dim}_{\mathbf{R}} Q(f)$ ? Clearly $\operatorname{dim}_{\mathbf{R}} Q(f)$ bounds the number of points near 0 of $f^{-1}(\varepsilon)$, for $\varepsilon$ near 0 . Are there perhaps arbitrarily small deformations $f_{1}$ of $f$ such that $f_{1}^{-1}(\varepsilon)$ has $\operatorname{dim}_{\mathrm{R}} Q(f)$ points near 0 for (some) small $\varepsilon$ ? Damon and Galligo show that the answer is "yes" for $n=2$, and in some other cases, and conjecture that it should always be "yes".

(3) Another subject that invites future work is the case of groundfields other than $\mathbf{R}$. I would propose that the degree at 0 of a finite polynomial map 
$f: K^{n} \rightarrow K^{n}$, where $K$ is an arbitrary field of characteristic 0 , be defined to be the equivalence class of the quadratic form $\langle,\rangle_{\varphi}$ on the local ring of $f$ at 0 which we will write $Q_{K}(f)$. It can be shown [Eisenbud-Levine] that the equivalence class of this form is independent of $\varphi$. This definition is "right" in the case $K=\mathrm{C}$, the complex numbers, since there the degree is equal to $\operatorname{dim}_{\mathbf{C}} Q_{\mathbf{C}}(f)$, which is the only invariant of \langle\rangle$_{\varphi}$. If $K=\mathbf{R}$, we have seen that the degree is the signature, the primary invariant of the form (the other invariant, the dimension of $Q_{\mathbf{R}}(f)$, gives the degree of the "complexification"; our notion of "degree $f$ " will of course always include the degree of the extension of $f$ to any bigger field).

There is really no reason to stick to fields of characteristic $\mathbf{0}$ for all this, though naturally one should not use the Jacobian for $J$ over a field of characteristic $p$ ! If $X_{1}, \ldots, X_{n}$ are coordinates in the source $K^{n}$, and $f_{1}, \ldots, f_{n}$ are the components of $f$ (which all must vanish at 0 ) then in the power series ring $K\left[\left|X_{1}, \ldots, X_{n}\right|\right]$ we may find elements $a_{i j}$ such that

$$
\sum_{j} a_{i j} X_{j}=f_{i}, \quad i=1, \ldots, n .
$$

It can be shown that if $f$ is finite at 0 then $\operatorname{det} a_{i j}=D$ has a nonzero residue in $Q_{K}(f)=K\left[\left|X_{1}, \ldots, X_{n}\right|\right] /\left(f_{1}, \ldots, f_{n}\right)$ generating the unique minimal ideal, and that over a field of characteristic $0, J=\operatorname{dim}_{K} Q(f) \cdot D$ in $Q(f)$, so that we may use $D$ in place of $J$. Again, the class of $\langle,\rangle_{\varphi}$ is uniquely determined by $Q(f)$ and $D$, at least as long as char $K \neq 2$.

The question is, does this idea of degree have some other interpretation (or usefulness), for example in cohomology theory, as is the case over $\mathbf{R}$ or $\mathbf{C}$.

\section{REFERENCES}

[Bass] H. Bass, On the ubiquity of Gorenstein rings, Math. Z. 82 (1963), 8-28.

[Beauville] A. Beauville, Une notion de residue en géométrie analytique, Sem. P. Lelong, (1969/1970) Lecture Notes in Math., vol. 205, Springer-Verlag, New York, 1971, pp. 183-203.

[Berger] R. Berger, Differenten regulärer Ringe, J. Reine Angew. Math. 214/215 (1964), $441-442$.

[Damon-Galligo] J. Damon and A. Galligo, A topological invariant for stable map germs, Invent. Math. 32 (1976), 103-132.

[Mather] J. Mather, Stability of $C^{\infty}$ mappings. III, Finitely determined map germs, Inst. Hautes Études Sci. Publ. Math. 35 (1968), 127-156.

[Milnor] J. Milnor, (1) Topology from a differentiable point of view, University Press of Virginia, Charlottesville, Virginia, 1965.

(2) Singular points of complex hypersurfaces, Princeton Univ. Press, Princeton, N. J., 1970.

[Serre] J.-P. Serre, ,Corps locaux, Hermann, Paris, 1968.

[Scheja-Storch], Über Spurfunktionen vollständigen Durchschnitten, J. Reine Angew. Math. 278/279 (1975), 174-189.

[Zakaljukin] V. M. Zakaljukin, Algebraic computability of the index of a singular point of a vector field, Funkcional Anal. i. Prilożen 6 (1972), 77-78.

[Eisenbud-Levine] D. Eisenbud and H. Levine, An algebraic formula for the degree of a $C^{\infty}$ map germ, Ann. of Math. (2) 106 (1977), 19-44.

Department of Mathematics, Brandeis University, Waltham, Massachusetrs 02154 